\section{Opioid and Benzodiazepine Use in Women With Breast Cancer: More Work on the Horizon}

\author{
Juan P. Cata, MD, and Daniel D. Kim, MD
}

I n 2017, the U.S. Department of Health and Human Services declared the opioid epidemic a public health emergency in response to the widespread use and misuse of opioids. It was estimated that in 2017 alone, almost 58 opioid prescriptions were written for every 100 Americans, which is equivalent to 190 million prescriptions per year. Since then, a collective effort from different medical and scientific societies and the National Institutes of Health has been established to understand, mitigate, and reduce unjustified patterns of opioid prescriptions in the United States and other countries. Although the rate of prescription opioid use has shown a decline, a less recognized healthcare problem is that between 1996 and 2013 the growth in use of benzodiazepines dramatically increased. Bachhuber et al $^{1}$ showed a $67 \%$ increase in filled benzodiazepine prescriptions, reaching 13.5 million in 2013. In the same year, a whopping increase in the amount of prescribed lorazepam indicated $3.6 \mathrm{~kg}$ equivalents of the drug per 100,000 adults. Because benzodiazepines are also highly addictive prescription medications, we can consider their current use and misuse as the "occult" epidemic in the United States. ${ }^{2}$

Patients with cancer are typically treated with opioids to ameliorate the suffering from painful conditions caused by tumors themselves or their treatments. Benzodiazepines are also commonly prescribed to patients with cancer to help with the psychological burden caused by diagnosis or to palliate symptoms such as nausea, which is usually related to the administration of chemotherapy agents. Opioids and benzodiazepines are also prescribed simultaneously in patients with cancer. However, studies demonstrate that the concomitant prescription of opioids and benzodiazepines increases the risk of emergency room visits or inpatient admission due to opioid overdose (adjusted odds ratio, 2.14; 95\% Cl, 2.05-2.24) and the risk of death in a dose-response manner (adjusted hazard ratio, 3.86; 95\% Cl, 3.49-4.26).3.4 Given the current evidence, a recently published ASCO guideline recommends caution be exercised with the concomitant use of opioids and benzodiazepines. ${ }^{5}$

In this issue of JNCCN, Sakamoto et $a^{6}{ }^{6}$ reported current rates of new and persistent use of opioids and benzodiazepines in a large cohort of women aged $\geq 66$ years diagnosed with breast cancer. Using the SEER-Medicare linked database, the authors found that $67.4 \%$ and $10.3 \%$ filled a new opioid or benzodiazepine prescription, respectively, during their cancer treatment journey, and $4 \%$ reported concomitant use. In a similar study using a shorter period of observation, Check et $\mathrm{al}^{7}$ reported a higher rate (10\%) of concurrent use of opioids and benzodiazepines in women with breast cancer. Together, these studies suggest that women aged $\geq 66$ years present a high risk of developing prolonged use of opioids and benzodiazepines.

Interestingly, Sakamoto et $a^{6}{ }^{6}$ found that surgery combined with adjuvant therapies was an independent risk factor for new opioid and benzodiazepine use or their concomitant prescription. It is well accepted that opioids are strong analgesics to relieve moderate-to-severe postoperative pain, and benzodiazepines are typically administered to relieve perioperative anxiety in patients with cancer. However, the data presented by Sakamoto et $a^{6}$ suggest that the perioperative period could be considered a trigger for medication misuse, development of tolerance, and persistent opioid and benzodiazepine use $\left(2.7 \%\right.$ and $11.6 \%$, respectively). ${ }^{6}$ It is also worth noting the authors'

See page 29 for related article.

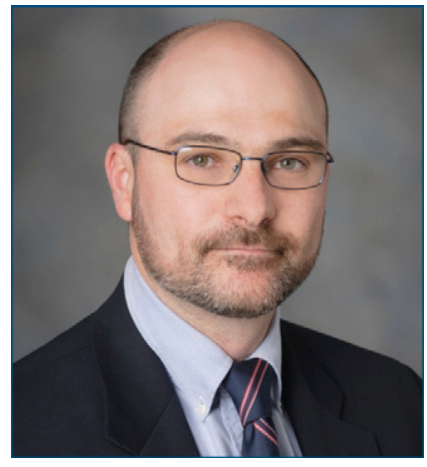

JUAN P. CATA, MD

Juan P. Cata, MD, is an Associate Professor in the Department of Anesthesiology and Perioperative Medicine at The University of Texas MD Anderson Cancer Center. Dr. Cata's research interest is focused on clinical predictors and mechanisms of chronic postsurgical pain and opioid use in patients with cancer.

doi: $10.6004 /$ jnccn.2020.7698

The ideas and viewpoints expressed in this commentary are those of the author and do not necessarily represent any policy, position, or program of NCCN 


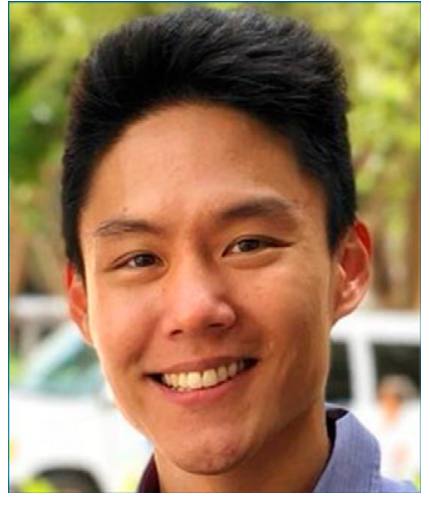

DANIEL D. KIM, MD

Daniel D. Kim, MD, is a cancer anesthesia fellow at The University of Texas MD Anderson Cancer Center, active member of the Outcomes Research Consortium, and past president of the Commission for pediatric anesthesia of the Sao Paulo State Anesthesia Society. findings indicating that persistent opioid use was reduced from 2008 to 2015, which highlights the efforts made to mitigate the opioid epidemic, and is in agreement with recently published data on opioid use in the United States. ${ }^{8,9}$ Conversely, there was no difference in persistent benzodiazepine use in the same period of investigation.

A comment is warranted about tramadol as a prescription analgesic. It is widely accepted that tramadol may present advantages over other opioids because of the 2 enantiomers' synergistic analgesic actions. ${ }^{10}$ Furthermore, tramadol is recommended by the WHO for step 2 in the ladder for cancer pain management, and is considered an opioid with a low risk of misuse or abuse in Europe and the United States. ${ }^{11,12}$ Unfortunately, however, the risks associated with tramadol use over long periods of consumption in opioid-naive patients with cancer have not been well established. Sakamoto et $a^{\mid}{ }^{6}$ demonstrated a significant association between tramadol use and a higher risk of opioid use at 3 and 6 months after breast cancer treatment, suggesting a potential misuse of the drug and other opioids. Interestingly, the results of a recently published cross-sectional study using the Taiwan National Health Insurance claims database indicate a 92.9-fold increase in tramadol prescriptions from 2002 to 2006 for patients without cancer. ${ }^{13}$ These studies highlight that changes in prescription patterns could make tramadol the preferred opioid for pain control after surgery in the United States.

In conclusion, while we wait for better evidence to indicate the potential risks associated with prolonged tramadol use in women with breast cancer or other malignancies, it would be prudent to not "trade one candy for another" that could potentially perpetuate the opioid epidemic. ${ }^{14}$ The finding that a significant number of women with breast cancer are persistently using benzodiazepines presents a new opportunity to develop strategies to reduce the unnecessary use of these agents.

Disclosures: The authors have disclosed that they have no financial interests, arrangements, or affiliations with the manufacturers of any products discussed in this article or their competitors.

Correspondence: Juan P. Cata, MD, Department of Anesthesiology and Perioperative Medicine, The University of Texas MD Anderson Cancer Center, Unit 409, 1515 Holcombe Boulevard, Houston, TX 77005. Email: JCata@mdanderson.org

\section{References}

1. Bachhuber MA, Hennessy S, Cunningham CO, Starrels JL. Increasing benzodiazepine prescriptions and overdose mortality in the United States, 1996-2013. Am J Public Health 2016;106:686-688.

2. Niles JK, Gudin J, Radcliff J, Kaufman HW. The opioid epidemic within the COVID-19 pandemic: drug testing in 2020 [published online October 8, 2020]. Popul Health Manag, doi: 10.1089/pop.2020.0230

3. Sun EC, Dixit A, Humphreys K, et al. Association between concurrent use of prescription opioids and benzodiazepines and overdose: retrospective analysis. BMJ 2017;356:j760.

4. Park TW, Saitz R, Ganoczy D, et al. Benzodiazepine prescribing patterns and deaths from drug overdose among US veterans receiving opioid analgesics: case-cohort study. BMJ 2015;350:h2698.

5. Paice JA, Portenoy R, Lacchetti $C$, et al. Management of chronic pain in survivors of adult cancers: American Society of Clinical Oncology clinical practice guideline. J Clin Oncol 2016;34:3325-3345

6. Sakamoto MR, Eguchi M, Azelby CM, et al. New persistent opioid and benzodiazepine use after curative-intent treatment in patients with breast cancer. J Natl Compr Canc Netw 2021;19:29-38

7. Check DK, Winn AN, Fergestrom N, et al. Concurrent opioid and benzodiazepine prescriptions among older women diagnosed with breast cancer. J Natl Cancer Inst 2020;112:765-768.

8. Cicero TJ, Ellis MS, Harney J. Shifting patterns of prescription opioid and heroin abuse in the United States. N Engl J Med 2015;373:1789-1790.

9. Wilson N, Kariisa M, Seth P, et al. Drug and opioid-involved overdose deaths - United States, 2017-2018. MMWR Morb Mortal Wkly Rep 2020;69:290-297.

10. Grond S, Sablotzki A. Clinical pharmacology of tramadol. Clinical Pharmacokinet 2004;43:879-923.

11. Iwanicki JL, Schwarz J, May KP, et al. Tramadol non-medical use in four European countries: a comparative analysis [published online October 20, 2020]. Drug Alcohol Depend, doi: 10.1016/j.drugalcdep.2020.108367

12. Reines SA, Goldmann B, Harnett M, Lu L. Misuse of tramadol in the United States: an analysis of the National Survey of Drug Use and Health 2002-2017. Subst Abuse 2020;14:1178221820930006.

13. Chen TC, Wang TC, Lin CP, et al. Increasing tramadol utilisation under strict regulatory control of opioid prescribing - a cross-sectional study in Taiwan from 2002 through 2016 [published online September 30, 2020]. J Formos Med Assoc, doi: 10.1016/j.jfma.2020.09.011

14. Farkas $A H$, Winn A, Pezzin LE, et al. The use and concurrent use of side effect controlling medications among women on aromatase inhibitors [published online October 26, 2020]. J Womens Health (Larchmt), doi: 10.1089/jwh.2020.8493 